\title{
Analysis of Vitamin D and Calcium Levels in Benign Paroxysmal Positional Vertigo
}

\author{
Gülşah Çıkrıkçı Işık1, Yunsur Çevik², Emine Emektar², Şeref Kerem Çorbacıoğlu² \\ ${ }^{1}$ Clinic of Emergency, Batman Region Community Hospital, Batman, Turkey \\ ${ }^{2}$ Clinic of Emergency, Health Science University Keçiören Training and Research Hospital, Ankara, Turkey
}

Cite this article as: Çıkrıkçı Işık G, Çevik Y, Emektar E, Çorbacıoğlu ŞK. Analysis of Vitamin D and Calcium Levels in Benign Paroxysmal Positional Vertigo. Eurasian J Emerg Med 2017; 16: 128-32

\begin{abstract}
Aim: The aim of the study was to evaluate the effects of vitamin D and calcium levels on the incidence and recurrence of benign paroxysmal positional vertigo (BPPV).

Materials and Methods: Sixty-four patients diagnosed with BPPV and 63 healthy volunteers who were age-sex matched and admitted to emergency department (ED) between October 1, 2014 and December 31, 2014 were included. Vitamin D and total calcium levels of blood samples collected from both groups upon admission were analyzed.

Results: The mean vitamin D level in the patient and control groups was $9.51 \pm 5.49 \mathrm{ng} / \mathrm{mL}$ and $11.02 \pm 9.62 \mathrm{ng} / \mathrm{mL}$, respectively. The mean total calcium level in the patient and control groups was $9.5 \pm 0.63 \mathrm{mg} / \mathrm{dL}$ and $9.41 \pm 0.49 \mathrm{mg} / \mathrm{dL}$, respectively. A significant difference was not detected between groups in terms of both parameters ( $p=0.992 ; p=0.345$, respectively). The mean vitamin D level in patients with a first episode was $9.91 \pm 5.81 \mathrm{ng} / \mathrm{mL}$ and in those who had similar symptoms earlier was $8.81 \pm 4.90 \mathrm{ng} / \mathrm{mL}$. The difference between groups was not significant $(\mathrm{p}=0.629)$.

Conclusion: There are studies showing low levels of vitamin D as a risk factor for incidence and recurrence of BPPV. However, in this study, no relationship was found between vitamin D and total calcium levels and BPPV incidence and recurrence. This result can be due to high frequency of vitamin D deficiency in the Turkish society. We also believe that because the study was conducted in winter, related to seasonal variability of vitamin D levels, the levels were even lower. However, for conclusive results, more comprehensive studies including year-round examinations are needed.
\end{abstract}

Keywords: Vitamin D, calcium, benign paroxysmal positional vertigo

\section{Introduction}

The most common and benign cause of vertigo, which is defined as the illusion of movement of the body or environment, is benign paroxysmal positional vertigo (BPPV). Although the pathophysiological process of the disease has not been fully clarified, currently, the widely accepted opinion is that the disease results from the accumulation of otoconia that are detached from the utricular macula in the semicircular canals and thereby sensitizing such canals to gravity (1). Otoconia crystals consist of a central nucleus mostly composed of organic glycoproteins with low calcium (Ca) levels and surrounding inorganic peripheral zones containing minerals mostly composed of calcium carbonate with high Ca levels.

Recent studies conducted to clarify the etiology have demonstrated that there may be an association between osteoporosis and Ca metabolism and the frequency of BPPV $(1,2)$. This is supported by the fact that BPPV is more common especially in postmenopausal women aged $>50$ years, in whom osteopenia and osteoporosis are common. Such studies have suggested that osteoporosis and accordingly the Ca metabolism may be a risk factor for developing BPPV by affecting particularly the peripheral zone in otoconia, which have a similar structure with bone tissue (3). The role of vitamin D, 
which is directly related to Ca metabolism and bone formation, in BPPV has been concerned and the vitamin D levels of BPPV patients have been investigated.

The primary objective of this study was to establish whether the vitamin D and total Ca levels of patients diagnosed with BPPV are lower compared to the normal population. Additionally, the secondary objective of our study was to determine whether there is a difference in vitamin $D$ and Ca levels of the patients with recurrent vertigo attacks compared to the control group and the patients who have experienced the first vertigo attack.

\section{Materials and Methods}

After receiving approval from the ethics committee of Keçiören Training and Research Hospital this study was conducted prospectively between October 1, 2014 and December 31, 2014, at an emergency service of a training and research hospital with a mean daily patient number of 750 .

The study included patients who presented to the emergency service with dizziness and were diagnosed with BPPV. The control group consisted of healthy subjects with demographic characteristics similar to the patient group.

The study inclusion criteria were patients aged $>18$ years, agreeing to participate in the study, and diagnosed with BPPV following the assessment in the emergency department.

The study exclusion criteria included patients $<18$ years of age; not agreeing to participate in the study; being pregnant; being diagnosed with central vertigo; having another existing disease that causes peripheral vertigo; having any disease, such as known osteoporosis that could affect vitamin D and Ca metabolism; or using any drugs such as Ca preparation or bisphosphonate that could affect vitamin D and Ca levels.

Patients who presented to the emergency department with dizziness were evaluated by emergency physicians. Detailed medical histories were obtained and physical examinations were performed and recorded into the study form for all the patients.

Benign paroxysmal positional vertigo was diagnosed based on the history and physical examination findings. The history was questioned in terms of the character, duration, emergence type, increasing and decreasing factors of the dizziness, and the accompanying symptoms as directive for BPPV. BPPV was considered primarily in patients describing dizziness that increased with head movements, emerged suddenly, or was short term but severe and decreased at rest. All patients were neurologically evaluated in detail, cerebral tests were performed, and the presence of ataxia was examined. Head-Thrust Test and Dix-Hallpike test were performed on patients to investigate the presence and characteristics of nystagmus. The presence of nystagmus that emerged after a latent period of time, lasted less than 1 minute, and exhausted with repetitive maneuvers, was considered as directive for BPPV diagnosis. Cranial tomography and diffusion magnetic resonance imaging (MRI) were conducted on the patients with suspected central pathology based on the history and neurological examination to eliminate potential pathologies. Patients with a detected central pathology were excluded from the study. A hearing test was performed on the patients describing fullness in the ear, loss of hearing, and tinnitus among those with suspected peripheral vertigo, again based on history and physical examination findings to eliminate Meniere's disease. The patients with detected Meniere's disease in the test were excluded from the study. Patients for whom the differential diagnosis of vertigo could not be established, despite all neurological examination, tests and imaging methods, were excluded from the study.

The patients diagnosed with BPPV based on history, physical examination, and other additional tests were divided into two groups as describing vertigo for the first time and describing recurrent vertigo. Patients who previously described one or more similar complaints were assessed in the recurrent vertigo group.

From all patients diagnosed with BPPV and the control group, consent was obtained at the emergency department admission, blood samples were collected, and the vitamin D and total Ca levels were studied immediately at the central biochemistry laboratory.

The total Ca level was studied with the photometric method using an AU 680 Beckman Coulter ${ }^{\circledR}$ kit. The normal level for total Ca was considered 8.5-10.2 mg/dL. The vitamin D level was studied with the chemiluminescence method using the DiaSorin Liaison ${ }^{\circledast}$ device, and the normal level was considered as $20-50 \mathrm{ng} / \mathrm{mL}$.

\section{Statistical analysis}

The data were analyzed using Statistical Package for Social Sciences (SPSS Inc.; Chicago, IL, USA) for Windows 16 software package. The compatibility of discrete and continuous numeric variables with normal distribution was analyzed using the Kolmogorov-Smirnov test. Descriptive statistics were expressed in mean \pm standard deviation or median (interquartile ration [IQR]: 25\%-5\%) for discrete and continuous variables, and categorical variables were expressed as number of cases and percentage.

The Mann-Whitney U-test was used to analyze whether there was a statistically significant difference for the non-normally distributed data of the peripheral vertigo and control group measurements. The Spearman's correlation test was used to examine whether there was a statistically significant correlation between age and vitamin $D$ and $C a$ measurements in the peripheral vertigo and the control groups. $\mathrm{P}<0.05$ was considered statistically significant for the results.

\section{Results}

In total, 64 patients diagnosed with BPPV were included in the study; 41 of them (64.1\%) had not experienced similar symptoms before, 16 of them (25\%) had an attack previously but did not have the diagnosis, and seven of them (10.9\%) were diagnosed with vertigo previously. Sixty-three healthy subjects were included in the study as the control group. Of the patients, 47 (73.4\%) were females and 17 (26.6\%) were males. In the patient group, the mean age was $56.19 \pm 13.46$ and $55.65 \pm 12$ years for female and male participants, respectively. Of the subjects in the control group, 45 (71.4\%) were females and 18 (28.6\%) were males; the mean age was $55.62 \pm 13.96$ and $59.56 \pm 13.66$ years for female and male participants, respectively. There was no difference in age and gender distribution between the two groups $(p=0.29$ and $\mathrm{p}=0.8$, respectively).

The median vitamin D value was $9.51 \mathrm{ng} / \mathrm{mL}$ (minimum-maximum [min-max]: 4-23.2) in the patient group and $7.8 \mathrm{ng} / \mathrm{mL}$ (IQR: 4.6-14.1) in the control group. The difference in vitamin $D$ levels between two groups was not statistically significant $(p=0.99)$. The median total Ca value was $9.5 \mathrm{mg} / \mathrm{dL}$ (min-max: 8.32-13) in the patient 
Table 1. Comparing vitamin D and total Ca levels in patient and control groups

\begin{tabular}{|c|c|c|}
\hline & Vit. D (ng/mL) & T.Ca (mg/dL) \\
\hline & Mean $\pm S D$ (min-max) & Mean $\pm S D(\min -\max )$ \\
\hline Patient & $9.51 \pm 5.49(4-23.2)$ & $9.5 \pm 0.63(8.32-13)$ \\
\hline Control & $11.02 \pm 9.62(4-56.8)$ & $9.41 \pm 0.49(8.28-10.36)$ \\
\hline$p$ & 0.992 & 0.345 \\
\hline
\end{tabular}

Table 2. Relationship between age and vitamin D and total Ca levels in the patient and control groups

\begin{tabular}{|c|c|c|c|c|c|c|c|c|}
\hline \multirow{2}{*}{} & \multicolumn{4}{|c|}{ Patient } & \multicolumn{4}{c|}{ Control } \\
\cline { 2 - 9 } & \multicolumn{2}{|c|}{ Vit. D } & \multicolumn{2}{|c|}{ T.Ca } & \multicolumn{2}{c|}{ Vit. D } & \multicolumn{2}{c|}{ T.Ca } \\
\cline { 2 - 9 } & $\mathrm{r}$ & $\mathrm{p}$ & $\mathrm{r}$ & $\mathrm{p}$ & $\mathrm{r}$ & $\mathrm{p}$ & $\mathrm{r}$ & $\mathrm{p}$ \\
\hline Age & -0.011 & 0.933 & -0.047 & 0.712 & 0.040 & 0.753 & -0.302 & 0.016 \\
\hline
\end{tabular}

Vit. D: vitamin D; T.Ca: total calcium; SD: standard deviation; $p$ value: $<0.05$ was considered statistically significant. $r$ value: correlation coefficient

Table 3. Relationship between vitamin D and total Ca levels in the patient and control groups

\begin{tabular}{|l|c|c|c|c|}
\hline \multirow{2}{*}{} & \multicolumn{2}{|c|}{ Patient } & \multicolumn{2}{c|}{ Control } \\
\cline { 2 - 5 } & \multicolumn{2}{|c|}{ Vit. D } & \multicolumn{2}{c|}{ Vit. D } \\
\cline { 2 - 5 } & $\mathrm{r}$ & $\mathrm{p}$ & $\mathrm{r}$ & $\mathrm{p}$ \\
\hline Total Ca & 0.089 & 0.486 & 0.088 & 0.493 \\
\hline
\end{tabular}

Vit. D: vitamin D; T.Ca: total calcium; SD: standard deviation; $p$ value: $<0.05$ was considered statistically significant. $r$ value: correlation coefficient

group and $9.43 \mathrm{mg} / \mathrm{dL}$ : (IQR: 9.12-9.68) in the control group. The difference in total Ca levels between the two groups was not statistically significant ( $p=0.34$; Table 1$)$.

The median vitamin D value was $6.8 \mathrm{ng} / \mathrm{mL}$ (IQR: $5.56-11.5$ ) in the recurrent BPPV group and $7.8 \mathrm{ng} / \mathrm{mL}$ (IQR: 4.6-14.1) in the control group. The difference in vitamin $D$ levels between the two groups was not statistically significant $(p=0.69)$. The median total $C a$ value was $9.65 \mathrm{mg} / \mathrm{dL}$ (IQR: 9.09-9.76) in the recurrent BPPV group and 9.43 $\mathrm{mg} / \mathrm{dL}$ (IQR: 9.12-9.68) in the control group. The difference in total Ca levels between the two groups was not statistically significant $(p=0.36)$.

The median vitamin D value was $8.2 \mathrm{ng} / \mathrm{mL}$ (IQR: $5.4-12.7$ ) and the median Ca level was $9.5 \mathrm{mg} / \mathrm{dL}$ (IQR: 9.08-9.70) in patients with the first BPPV attack. When these values were compared with those of the recurrent BPPV patients, no statistically significant difference was found $(p=0.99$ for vitamin $D ; p=0.34$ for $\mathrm{Ca}$ ).

There was no significant relationship between age and vitamin D and Ca levels in both groups ( $p>0.05$ ). In the patient group, a statistically significant correlation could not be found between age and vitamin $D$ and Ca levels ( $p=0.99, r=-0.01 ; p=0.71, r=-0.04)$. In the control group, the relationship between age and vitamin $D$ levels was examined; however, no significant correlation was established $(p=0.75, r=0.04)$. An inverse statistical correlation was found between age and Ca levels; however, such a correlation was weak ( $p=0.01$, $r=-0.30$; Table 2).

The correlation between vitamin D and Ca levels was also evaluated in the patient and control groups, but a statistically significant correlation could not be established $(p=0.48, r=0.08 ; p=0.49, r=0.08$; Table 3).

\section{Discussion}

It is important to clarify the etiology of BPPV, which is the most common vestibular disease, for effective treatment and recurrence prevention. The present study examined vitamin $\mathrm{D}$ and total $\mathrm{Ca}$ levels in patients diagnosed with BPPV, who presented to the emergency service with dizziness. No relationship was established between these values and the disease incidence and recurrence rate.

Firstly, the pathophysiology of the disease should be understood to associate BPPV with vitamin D. The vestibular part of the membranous labyrinth consists of three semicircular canals as anterior, posterior, and horizontal, and two otoliths as utricle and saccule; the source of the calcium carbonate crystals (otoconia) that are responsible for BPPV is the macula of the saccule (4). Canalithiasis refers to the displacement of otoconia located within the gelatinous membrane in the macula into the semicircular canals, whereas cupulolithiasis defines the adherence of these particles to the cupula of the semicircular canals. These two conditions are the mechanisms considered responsible for BPPV pathophysiology (5).

Otoconia consist of a central part with a regular and dense structure and a peripheral part with an irregular and porous structure (6). Calcium carbonate, the main inorganic component of otoconia, is present more intensely in the peripheral zone (1). Since BPPV is more common in postmenopausal women aged $>50$ years, it has been considered that osteopenia and osteoporosis may be risk factors for developing BPPV by affecting particularly the peripheral zone in otoconia, which have a structure similar to the bone tissue (3).

To establish this relationship, Vibert et al. (3) conducted a study on rats and found distinctively reduced density and increased volume of otoconia in osteoporotic rats compared to the control group. In a similar vein, Jeong et al. (7) conducted a study with 209 patients diagnosed with idiopathic BPPV and established that the presence of osteopenia or osteoporosis alone increased the risk of BPPV after excluding other variables, such as age, gender, alcohol use, smoking, and hyperphosphatemia. The study by Yamanaka et al. (8) indicated that the osteoporosis incidence in BPPV patients was similar to the rest of the population, but the BPPV patients with osteoporosis had a distinctively higher recurrence incidence compared to the BPPV patients with normal bone mineral density, that is, the presence of osteoporosis posed a risk for BPPV recurrence.

Nevertheless, recently published studies related to this topic have not defended the same idea with the older studies. The study by Karataş et al. (9) indicated that prevalence of osteoporosis and vitamin $D$ deficiency is reasonably high in the general population, and the coexistence of BPPV with osteoporosis and vitamin D deficiency is coincidental. In a similar vein, Kahraman et al. (10) indicated that vitamin D deficiency and decreased ionized Ca level may be a risk for BPPV, not only in patients with osteoporosis but also in all patients. 
After several studies have demonstrated the relationship between BPPV and osteopenia/osteoporosis, research is increasingly directed toward the role of vitamin D in BPPV, which is directly related to $\mathrm{Ca}$ and phosphorus metabolism and thereby bone formation. The study by Jeong et al. established lower serum levels of vitamin D in idiopathic BPPV patients regardless of age, gender, body mass index, hypertension, diabetes, proteinuria, regular exercise, and reduced bone mineral density (2). Buki et al. (1) found the serum levels of vitamin D in BPPV patients similar to the rest of the population, but showed that such levels were further lower in patients with recurrent BPPV compared to those with the first BPPV attack. Another study was conducted by Talaat et al. (11), which compared bone mineral density and vitamin $D$ levels in recurrent and non-recurrent BPPV patients. Based on the data, the study found bone mineral density associated with both development and recurrence of BPPV. It was suggested that low vitamin D levels were associated only with BPPV development, but very low levels had an effect on disease recurrence (11).

After several studies have shown correlation between vitamin D deficiency and development and the recurrence of BPPV, whether the treatment of severe vitamin D deficiency could affect the recurrence rate of BPPV was questioned. The study by Talaat et al. (12) established that improvement of serum 25-hydroxyvitamin D3 levels is associated with substantial decrease in the recurrence of BPPV. Similarly, Sheikhzadeh et al. (13) indicated in their study that the correction of vitamin D deficiency in BPPV provides additional benefit to rehabilitation therapy (Epley maneuver) regarding the duration of improvement.

The present study also found a lower mean vitamin D level in the patient group compared to the control group; however, such a difference was not statistically significant, unlike the literature $(p=0.992)$. To determine the effect of vitamin $D$ levels on recurrence, the patient group was divided into two subgroups: those with the first vertigo attack and those with recurrent complaints; however, no significant difference was found in vitamin $D$ levels between these subgroups $(p=0.345)$. This may have resulted from the fact that vitamin D deficiency is currently a highly common issue in the Turkish population.

Vitamin D deficiency is a common health issue around the world. A study that investigated the vitamin D levels in Europe and Asia reported that Middle Eastern countries, including Turkey, have very low vitamin D levels (14). The study by Ciğerli et al. (15), which was conducted in Turkey, examined 2488 adult outpatients and found the mean vitamin $D$ level to be $17.4 \pm 11.5 \mathrm{ng} / \mathrm{mL}$. The study showed that $24 \%$ of the patients had vitamin D deficiency, $66 \%$ had vitamin $D$ inadequacy, and the vitamin D inadequacy increased, particularly during the autumn and winter months. The study by Karagüzel et al. (16) investigated vitamin D levels in school-going children and similarly demonstrated that the prevalence of vitamin D inadequacy was $93 \%$ in the autumn, whereas it regressed to $71 \%$ in the spring. Atlı et al. (17) conducted a study with an elderly population and reported that vitamin D inadequacy was more common in older women, and the vitamin D levels were inversely proportional to age.

Besides age and seasonal factors, several factors, such as gender, skin color, dressing habits, food habits, using supplements, and body mass index, influence vitamin D levels (10). Our study was conducted between October and December, and most of the groups consisted of females and the mean age was higher; all of these are among the factors that cause the vitamin D levels to be low.
Due to the effects of vitamin D on the Ca metabolism, Parham et al. (18) examined the relationship between the presence of BPPV and vitamin $\mathrm{D}$ and serum ionized $\mathrm{Ca}$, but could not establish any link. We also evaluated this relationship in our study. Similarly, we could not find any significant difference in total $\mathrm{Ca}$ levels between the patient and control groups. Additionally, we could not establish any significant relationship between vitamin $\mathrm{D}$ and Ca levels possibly because the total Ca levels are affected by several factors other than vitamin D.

\section{Conclusion}

A statistically significant difference was not found in vitamin D and total Ca levels between the patients diagnosed with BPPV in the emergency service and the control group.

This may be because the vitamin $D$ inadequacy is very common even in healthy individuals in the Turkish population. Furthermore, given the seasonal changes in vitamin $D$ levels, it is an important limitation that we have conducted the study between October and December.

To reach a specific conclusion, long-term and broader studies that would include the entire year are needed.

Ethics Committee Approval: Ethics committee approval was received for this study from the ethics committee of Keçiören Training and Research Hospital.

Informed Consent: Written informed consent was obtained from patients who participated in this study.

Peer-review: Externally peer-reviewed.

Conflict of Interest: No conflict of interest was declared by the authors.

Financial Disclosure: The authors declared that this study has received no financial support.

\section{References}

1. Büki B, Ecker $M$, Jünger $H$, Lundberg $Y W$. Vitamin $D$ deficiency and benign paroxysmal positioning vertigo. Med. Hypotheses 2013; 80: 201-4. [CrossRef]

2. Jeong SH, Kim JS, Shin JW, Kim S, Lee H, Lee AY, et al. Decreased serum vitamin $D$ in idiopathic benign paroxysmal positional vertigo. J Neurol 2013; 260: 832-8. [CrossRef]

3. Vibert D, Sans A, Kompis M, Travo C, Mühlbauer RC, Tschudi I, et al. UItrastructural changes in otoconia of osteoporotic rats. Audiol Neurootol 2008; 13: 293-301. [CrossRef]

4. Xiang-Dong G. Bening paroxysmal positional vertigo. J Neurosci Rural Pract 2011; 2: 109-10. [CrossRef]

5. Parnes LS, Agrawal SK, Atlas J. Diagnosis and management of benign paroxysmal positional vertigo. CMAJ 2003; 169: 681-93.

6. Walther LE, Blödow A, Bloching MB, Buder J, Corrillo-Cabrera W, Roseeva $E$, et al. The inner structure of human otoconia. Otol Neurotol 2014; 35 : 686-94. [CrossRef]

7. Jeong SH, Choi SH, Kim JY, Koo JW, Kim HJ, Kim JS. Osteopenia and osteoporosis in idiopathic bening positional vertigo. Neurology 2009; 72: 1069-76. [CrossRef]

8. Yamanaka T, Shirota S, Sawai Y, Murai T, Fujita N, Hosoi H. Osteoporosis as a risk factor for the recurrence of bening paroxysmal positional veritgo. Laryngoscope 2013; 123: 2813-6. [CrossRef]

9. Karataş A, Acar YG, Yüce T, Hacı C, Cebi IT, Salviz M. Association of Benign Paroxysmal Positional Vertigo with Osteoporosis and Vitamin D 
Deficiency: A Case Controlled Study. J Int Adv Otol 2017. doi: 10.5152/ iao.2016.2640 [CrossRef]

10. Kahraman SS, Ozcan O, Arli C, Ustun I, Erduran R, Akoglu E, Gokce C. Calcium Homeostasis During Attack and Remission in Patients With Idiopathic Benign Paroxysmal Positional Vertigo. Otol Neurotol 2016; 37: 1388-92. [CrossRef]

11. Talaat HS, Abudahied G, Talaat AS, Sbdelaal MS. Low bone mineral density and vitamin $D$ deficiency in patient with bening positional paroxysmal vertigo. Eur Arch Otorhinolaryngol 2015; 272: 2249-53. [CrossRef]

12. Talaat HS, Kabel AM, Khaliel LH, Abuhadied G, El-Naga HA, Talaat AS. Reduction of recurrence rate of benign paroxysmal positional vertigo by treatment of severe vitamin D deficiency. Auris Nasus Larynx 2016; 43: 237-41. [CrossRef]

13. Sheikhzadeh M, Lotfi Y, Mousavi A, Heidari B, Monadi M, Bakhshi E. Influence of supplemental vitamin $D$ on intensity of benign paroxysmal positional vertigo: A longitudinal clinical study. Caspian J Intern Med 2016; 7: 93-8.
14. Lips P. Vitamin D status and nutrition in Europe and Asia. J Steroid Biochem Mol Biol 2007; 103: 620-5. [CrossRef]

15. Cigerli O, Parildar H, Unal AD, Tarcin O, Erdal R, Demirag NG. Vitamin $D$ deficiency is a problem for adult out-patients? A üniversity hospital sample in Istanbul, Turkey. Public Health Nutr 2013; 16: 1306-13. [CrossRef]

16. Karagüzel G, Dilber B, Çan G, Ökten A, Değer O, Holick MF. Seasonal vitamin $D$ status of healthy schoolchildren and predictors of low vitamin $D$ status. J Pediatr Gastroenterol Nutr 2014; 58: 654-60. [CrossRef]

17. Atlı T, Güllü S, Uysal AR, Erdogan G. The prevalence of Vitamin D deficiency and effects of ultraviolet light on Vitamin D levels in elderly Turkish Population. Arch Gerentol Geriatr 2005; 40: 53-60. [CrossRef]

18. Parhan K, Leonard G, Feinn RS, Lafreinere D, Kenny AM. Prospective clinical investigation of the relationship between idiopathic bening paroxysmal positional vertigo and bone turnover: a pilot study. Laryngoscope 2013; 123: 2834-9.[CrossRef] 